clinicians on health economic grounds in patients after three hospital admissions for acute hypercapnic respiratory failure. Previous systematic reviews of domiciliary NIV have been limited in scope and required updating.

Methods Standard systematic review methods were used for identifying relevant clinical and cost-effectiveness studies of any appropriate design assessing NIV compared to usual care, or comparing different types of NIV. Risk of bias was assessed and checked. Primary effectiveness outcomes (mortality, hospitalisations, exacerbations and quality-of-life) were combined using random effects meta-analysis. Results were grouped into patients given NIV within 6 weeks of a hospital admission requiring inpatient NIV and those given NIV when stable.

Results Thirty controlled effectiveness studies were identified reporting a variety of outcomes, together with 65 uncontrolled studies. Benefit from NIV in terms of survival and hospital admissions in controlled studies was variable, and where present appeared most marked in post-hospital patients (based on limited evidence). For more stable patients, a modest volume of evidence found no benefit from NIV for survival and some nonsignificant beneficial trends for hospitalisations and quality-of life. No conclusions could be drawn regarding potential benefit from different types of NIV due to limited study sizes and heterogeneity.

Conclusions Domiciliary NIV has greatest effect when used after a hypercapnic exacerbation and might improve hospitalisation rates and mortality in this group of patients. There is no benefit if used in stable, normocapnic patients.

This abstract summarises independent research funded by the National Institute for Health Research (NIHR) under its HTA Programme (Ref 11/27/01). The views expressed are those of the author (s) and not necessarily those of the NHS, the NIHR or the Department of Health.

\section{P301 A LARGE RETROSPECTIVE EVALUATION OF DOMICILIARY AND OUTPATIENT INITIATION OF HOME MECHANICAL VENTILATION}

JM Palmer, NR Ward, JC Robinson, B Kathiresan, PD Hughes. Derriford Hospital, Plymouth, UK

\subsection{6/thoraxjn-2014-206260.419}

Introduction and objectives Home Mechanical Ventilation (HMV) for patients with chronic ventilatory failure (CVF) often requires hospital admission for initiation of treatment. There are limited data evaluating the efficacy, efficiency and safety of initiating HMV in the domiciliary setting. Our centre has undertaken over 200 'home set-ups' and we have evaluated outcomes in these patients.

\begin{tabular}{|c|c|c|}
\hline & Mean (SD) & Range \\
\hline Vital Capacity (L) & $2.1(1.2)$ & $0.45-5.5$ \\
\hline $\mathrm{pH}$ & $7.39(0.04)$ & $7.25-7.48$ \\
\hline Arterial $\mathrm{pCO} 2(\mathrm{kPa})$ & $7.0(1.6)$ & $3.2-14.5$ \\
\hline Arterial p02 (kPa) & $9.2(2.0)$ & $5.8-18.9$ \\
\hline Bicarbonate (mmols/L) & $29.5(5.8)$ & $21-51$ \\
\hline
\end{tabular}

Methods Patients with CVF who had HMV initiated in the domiciliary or outpatient setting were identified from our hospital database and data were retrospectively collected from their hospital records.

Results 214 patients with CVF were set-up at home between 2004 to 2013. Notes were available for 193 (90\%) patients, mean (SD) age 59 (14) years, 63\% male. The majority of patients had Motor Neuron Disease (MND)(30\%) or obesity related respiratory failure (23\%). Baseline lung function and arterial blood gas parameters are shown in Table 1.

178 (92\%) patients had HMV initiated in their home; 15 attended the outpatient clinic for set-up. Three patients subsequently required hospital admission to support adaptation to HMV.

Following initiation, 135 (70\%) patients were assessed as compliant with HMV, defined as $>4$ h self-reported use each night. Patients with MND had the lowest compliance rate with only $30(52 \%)$ achieving this usage. If those with MND are excluded, overall compliance was $77 \%$ which is similar to our inpatient initiated HMV compliance rate of $83 \%(n=224)$ and to case series reported by other centres.

Patients with few symptoms of nocturnal hypoventilation had a lower compliance rate $(55 \%)$ than more symptomatic patients (71\%).

In patients who were compliant with HMV, mean (SD) time until $>4$ h use per night was 27 (60) days, but 33 (17\%) patients achieved this usage after the first night. Those who became compliant with HMV had a mean of 2.9 (3.2) home visits and 1.4 (1.8) phone calls each.

Conclusion Establishing HMV in the domiciliary and outpatient setting can be effectively and safely achieved, even in patients with marked nocturnal hypoventilation. Apart from patients with MND or those who are minimally symptomatic, 'home setup' of HMV does not appear to affect compliance significantly.

\section{P302 HOME MECHANICAL VENTILATION: HAS VENTILATOR TECHNOLOGY SURPASSED OUR ABILITY TO CARE FOR SOME PATIENTS IN A COMMUNITY SETTING?}

D Worsley, F Telford, DB Land. University Southampton NHS FoundationTrust, Southampton, UK

10.1136/thoraxjnl-2014-206260.420

Introduction Home mechanical ventilation (HMV) for patients with chronic respiratory failure is a growing therapeutic modality that can reduce morbidity and mortality. ${ }^{1} \mathrm{HMV}$ may be complex to establish and requires a clear care pathway from acute to community services. The aim was to ascertain factors influencing inpatient length of stay $(\mathrm{LoS})$ and mortality in individuals requiring HMV who were unable to use the device independently.

All HMV was initiated in a respiratory high dependency unit (RHDU) in a university hospital.

Method A retrospective analysis of medical notes was conducted for all patients initiated on HMV between September 2012 and September 2013. Patients who were unable to manage the device independently were identified. Data collected included: admission data, social history, primary diagnosis, date deemed medically fit, readmission to RHDU, bed days post medically fit (section 5), reasons for delayed discharge and outcome.

LoS and bed day cost were calculated based on trust finance data for level 1 and 2 beds. 